\title{
Tolerance and efficacy of palliative radiotherapy for advanced pancreatic cancer: A retrospective analysis of single-institutional experiences
}

\author{
EDYTA WOLNY-ROKICKA ${ }^{1,2}$, KRZYSZTOF SUTKOWSKI $^{3}$, ALEKSANDRA GRZĄDZIEL $^{4}$, \\ ŻANETA DORSZ ${ }^{2}$, ANDRZEJ TUKIENDORF ${ }^{5}$, JAKUB LIPIŃSKI ${ }^{6}$ and JERZY WYDMAŃSKI ${ }^{2}$
}

\author{
${ }^{1}$ Department of Radiotherapy, Lubuski Center of Oncology, Regional Hospital in Zielona Góra, 65-001 Zielona Góra; \\ ${ }^{2}$ Department of Radiotherapy, Center of Oncology, Maria Sklodowska-Curie Memorial Institute, Gliwice Branch, 44-101 Gliwice; \\ ${ }^{3}$ First Department and Clinic of General, Gastroenterological and Endocrinological Surgery, Wrocław Medical University, \\ 50-369 Wrocław; Departments of ${ }^{4}$ Medical Physics and ${ }^{5}$ Epidemiology and Silesia Cancer Registry, Center of Oncology, \\ Maria Sklodowska-Curie Memorial Institute, Gliwice Branch, 44-101 Gliwice; ${ }^{6}$ University of Zielona Góra, \\ Faculty of Computer, Electrical and Control Engineering, 65-001 Zielona Góra, Poland
}

Received May 22, 2015; Accepted March 24, 2016

DOI: $10.3892 / \mathrm{mco} .2016 .851$

\begin{abstract}
The present study was conducted to investigate hypofractionated radiotherapy (RT) in patients with locally advanced or metastatic adenocarcinoma of the pancreas. A total of 31 patients were enrolled in this study, 26 of whom had locally advanced (M0) pancreatic cancer and 5 had metastatic (M1) disease. The patients were treated with palliative RT (6-30 Gy in 1-10 fractions over a period of 1 day-2 weeks). Treatment-related toxicity was classified according to the Common Terminology Criteria for Adverse Events, version 3.0. Early mild toxicity was observed. A total of 17 patients $(55 \%)$ achieved good pain control without pharmacological therapy, and 12 patients $(39 \%)$ reduced their use of analgesics; in the remaining 2 patients $(6 \%)$, there was no change in analgesic use. Late high-grade (>3) toxicity was not observed. The average survival time for the 31 patients was 9 months. The 1-year overall survival rate was $16 \%$. Palliative RT was well-tolerated and was able to prolong the survival time. The majority of the patients achieved better pain control with palliative RT.
\end{abstract}

\section{Introduction}

Pancreatic exocrine tumours are fatal in the majority of the patients, due to late diagnosis and poor response to combined

Correspondence to: Dr Edyta Wolny-Rokicka, Department of Radiotherapy, Lubuski Center of Oncology, Regional Hospital in Zielona Góra, Zyty 26, 65-001 Zielona Góra, Poland

E-mail: edyta.wolny@gmail.com

Key words: pancreatic cancer, palliative radiotherapy, pain, hypofractionated radiotherapy, advanced pancreatic cancer treatment. Patients with locally advanced unresectable or metastatic pancreatic cancer represent a clear majority among all patients with pancreatic cancer (1-4).

Pancreatic cancer is the fourth leading cause of cancer-related mortality, with a 5-year overall survival (OS) rate of $6 \%$ (3-7). Cancers that are detected incidentally are likely to be treated surgically, with or without adjuvant therapy. However, the median survival time is limited to 11-23 months and the 5-year survival rate is $<20 \%(2,4-6)$.

The majority of the patients present with incurable disease and several experience rapid clinical deterioration, without any improvement over several decades $(7,8)$.

Determining whether a tumour is resectable is not well reflected by TNM system staging, as demonstrated by the wide range of survival figures reported for each stage.

To be determined as resectable, tumours must show no evidence of extrapancreatic disease or direct tumour extension to the celiac axis and superior mesenteric artery. However, the evidence of non-obstructive superior mesenteric-portal vein confluence does not always preclude tumour resection.

Complete surgical resection is the only potentially curative treatment available.

For $\sim 35-40 \%$ of the patients who have locally advanced disease at the time of diagnosis, chemoradiation (CRT) is one of the most common treatments (9). CRT is an effective palliative treatment, although it has notable limitations. Studies have shown improved median survival times in patients treated with CRT compared with either chemotherapy (6) or RT alone. Patients with locally advanced disease may present with major local symptoms.

Optimal symptomatic treatment may play a key role in the management of metastatic disease. This may require stenting or bypass surgery for obstructive jaundice or gastric outlet obstruction. The most common symptom from local extension is pain, which may significantly affect the patient's quality of life. RT is used to control these symptoms, as was reported in locally advanced gastric cancer $(10,11)$. 
Table I. Patient and tumour characteristics in 31 patients.

\begin{tabular}{|c|c|}
\hline Characteristics & Patient no. (\%) \\
\hline \multicolumn{2}{|l|}{ Gender } \\
\hline Male & $18(58)$ \\
\hline Female & $13(42)$ \\
\hline \multicolumn{2}{|l|}{ Age, years } \\
\hline Median (range) & $57(35-77)$ \\
\hline $70-79$ & $8(26)$ \\
\hline $60-69$ & $8(26)$ \\
\hline$<60$ & $15(48)$ \\
\hline \multicolumn{2}{|c|}{ ECOG performance status } \\
\hline 0 & $3(10)$ \\
\hline 1 & $10(32)$ \\
\hline 2 & $13(42)$ \\
\hline 3 & $5(16)$ \\
\hline \multicolumn{2}{|l|}{ Tumour location } \\
\hline Head & $22(71)$ \\
\hline Body-tail & $9(29)$ \\
\hline \multicolumn{2}{|c|}{ Maximum tumour diameter, $\mathrm{cm}$} \\
\hline Median (range) & $5.12(2.2-11)$ \\
\hline \multicolumn{2}{|l|}{ Metastases } \\
\hline M0 & $26(84)$ \\
\hline M1 & $5(16)$ \\
\hline \multicolumn{2}{|c|}{ Histological diagnosis } \\
\hline Adenocarcinoma & $29(94)$ \\
\hline Others & $2(6)$ \\
\hline \multicolumn{2}{|l|}{ Differentiation } \\
\hline High & $14(45)$ \\
\hline Moderate & $3(10)$ \\
\hline Poor & $2(6)$ \\
\hline Not defined & $12(39)$ \\
\hline \multicolumn{2}{|l|}{ Percent weight loss } \\
\hline$<10 \%$ & $21(68)$ \\
\hline No loss & $10(32)$ \\
\hline \multicolumn{2}{|c|}{ Presenting symptoms prior to $\mathrm{RT}^{\mathrm{a}}$} \\
\hline Jaundice & $2(6)$ \\
\hline Abdominal pain & $21(68)$ \\
\hline Fatigue & $7(23)$ \\
\hline Diarrhoea & $10(32)$ \\
\hline Back pain & $10(32)$ \\
\hline
\end{tabular}

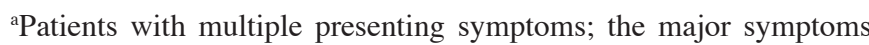
are listed. ECOG, Eastern Cooperative Oncology Group; M0, locally advanced; M1, metastatic; RT, radiotherapy.

\section{Patients and methods}

Patient population. The candidate subjects for this retrospective study were 31 patients with histologically proven unresectable pancreatic cancer registered at the Center of Oncology, Maria Sklodowska-Curie Memorial Institute
Table II. Treatment-related adverse events in 31 patients.

\begin{tabular}{lc}
\hline Grade of adverse events & Patient no. $(\%)$ \\
\hline Nausea & \\
G1 & $4(13)$ \\
G2 & $6(19)$ \\
G3-5 & $0(0)$ \\
Vomiting & \\
G1 & $2(6.5)$ \\
G2 & $3(10)$ \\
G3-5 & $0(0)$ \\
Diarrhoea & \\
G1 & $3(10)$ \\
G2 & $0(0)$ \\
G3-5 & $0(0)$ \\
Fatigue & \\
G1 & $7(23)$ \\
G2 & $0(0)$ \\
G3-5 & $0(0)$ \\
Abdominal pain & \\
G1 & $17(55)$ \\
G2 & $12(39)$ \\
G3, 4, 5 & $0(0)$ \\
\hline
\end{tabular}

(Gliwice, Poland) from November, 2000 to November, 2007. We included patients with stage III pancreatic cancer at the time of diagnosis, based on the 6th American Joint Committee on Cancer staging guidelines (12). The median age of the patients was 57 years (range, 35-77 years) and the median Eastern Cooperative Oncology Group performance status score was 2 (range, $0-3)$. The patients in the study included 18 men (58\%) and 13 women $(42 \%)$. The characteristics of the patients and tumours are summarised in Table I. The initial palliative treatment option for all the patients was chemotherapy based on 5-fluorouracil (5-FU) alone, or 5-FU with gemcitabine for the 5 patients with metastasis. Among the patients treated with chemotherapy for palliation, single-agent 5-FU was the most common treatment.

The primary endpoints of the study were OS, treatment-related toxicity and an estimated intensity of pain correlated with pancreatic cancer.

$R T$. Three-dimensional computer planning was used in all the cases. All the patients underwent CT scans (X-vision, Somatom; Siemens Inc., Munich, Germany) and the Eclipse system (Varian Medical Systems, Palo Alto, CA, USA) was used for RT treatment planning. The clinical target volume (CTV) included the primary tumour - whole pancreatic body, as detected by CT scans. The planning target volume was defined as the CTV plus $10-\mathrm{mm}$ margins in all directions. The two- (anterior and posterior) or three-field techniques (anterior, posterior and oblique lateral field) were used. RT was delivered with 20-MV X-rays. A total dose of 6-30 Gy was delivered in 1-10 fractions over a period of 1 day-2 weeks. 
Table III. Multiparameter analysis of prognostic factors affecting 1-year and overall survival rates.

\begin{tabular}{lccc}
\hline & & \multicolumn{2}{c}{ P-value } \\
\cline { 3 - 4 } Prognostic factors & Patient no. & 12-month & All-month \\
\hline Gender & & 0.65 & 0.49 \\
$\quad$ Male & 18 & & \\
Female & 13 & & \\
Age & & 0.02 & 0.02 \\
Location of tumour & & & \\
Head & 22 & 0.13 & 0.14 \\
Body-tail & 9 & 0.94 & 0.89 \\
Tumour diameter & & 0.15 & 0.14 \\
Weight loss & & & \\
$>10 \%$ & 21 & & 0.41 \\
No loss & 10 & 0.32 & \\
Metastases & & & 0.87 \\
M0 & 26 & 0.91 & \\
M1 & 5 & 0.26 & \\
Total dose (TD) & & & \\
Mean, 22.7 Gy & & & \\
Range, 6-30 Gy & & & \\
\hline
\end{tabular}

M0, locally advanced; M1, metastatic.

One patient $(3 \%)$ received $6 \mathrm{~Gy}$ in a single dose as hypofractionated treatment, 1 patient $(3 \%)$ received $11 \mathrm{~Gy}$ in 5 fractions, 3 patients $(10 \%)$ received $18 \mathrm{~Gy}$ in 6 fractions, 7 patients $(23 \%)$ received 20 Gy in 5 fractions and 19 patients $(61 \%)$ received 30 Gy in 10 fractions. We used two opposite fields in 22 patients $(71 \%)$ and three oblique fields in 9 patients (29\%). The V50\% of the liver was limited to $30 \mathrm{~Gy}$ and the V30\% of both kidneys was limited to $20 \mathrm{~Gy}$.

Toxicity criteria and tumour response. The treatment-related toxicities were classified according to the Common Terminology Criteria for Adverse Events, version 3.0 (13). Nausea, vomiting, diarrhoea, leukopaenia, granulocytopaenia, lymphocytopaenia and thrombocytopaenia were assessed weekly.

The pain intensity was assessed after 4 weeks of RT using a visual analogue scale. The tumour response was assessed based on the CT scans.

Statistical analysis. The OS was calculated from the first day of RT. The survival curves were constructed using the Kaplan-Meier method. Statistical analyses of categorical variables were performed using the arithmetic mean coefficient of dominance. The association between survival and one or more covariates was examined using the Cox proportional hazards regression model. The risk of early death was estimated using the hazard ratio (HR).

Two-sided P-values of $<0.05$ were considered significant. All analyses were performed using the $\mathrm{R}$ 2.11.1 programme for Windows XP (www.r-project.org).
Table IV. Single-parameter analysis of prognostic factors affecting overall survival rate.

\begin{tabular}{lcc}
\hline Prognostic factors & Patient no. & $\begin{array}{c}\text { OS } \\
\text { P-value }\end{array}$ \\
\hline Gender & 18 & 0.03 \\
Male & 13 & \\
Female & & 0.02 \\
Age & & \\
Location of tumour & 22 & 0.03 \\
Head & 9 & 0.14 \\
Body-tail & & 0.03 \\
Tumour diameter & & \\
Weight loss & 21 & 0.02 \\
$>10 \%$ & 10 & \\
No loss & & 0.15 \\
Metastases & 26 & \\
M0 & 5 & \\
M1 & & \\
Total dose (TD) & & \\
Mean, 22.7 Gy & & \\
Range, 6-30 Gy & & \\
\hline
\end{tabular}

OS, overall survival; M0, locally advanced; M1, metastatic.

\section{Results}

Tolerance and response to treatment. We observed an absence of RT interruptions, lack of hospitalisation due to toxic reactions and an absence of severe toxicity in patients undergoing palliative RT. Palliative RT was well-tolerated, with only 9 patients (29\%) requiring treatment for toxicity, which primarily included nausea and vomiting in patients with grade 2 (G2) events. The predominant G1 adverse events were abdominal pain and fatigue (Table II).

The pain intensity was evaluated prior to initiation and 1 month after RT, and the analgesic drug therapy was adjusted until a 0-3 pain score was reached (WHO).

A total of 17 patients (55\%) achieved good pain control (G1) without pharmacological therapy, 12 patients $(39 \%)$ reduced their use of analgesics $(\mathrm{G} 2)$, and in the remaining 2 patients $(6 \%)$ there was no change in analgesic use (G3).

Survival and pattern of failure. The median survival time for all 31 patients was 5 months (range, 1-53 months). The 1 -year OS rate was $16.13 \%$ (Fig. 1). An important positive predictor of survival in single- and multiparameter analyses was age $(\mathrm{P}=0.02)$. However, in the single-parameter analysis, the positive predictors of survival were male gender $(P=0.03)$ (Fig. 2), location of the tumour in the pancreatic head $(\mathrm{P}=0.03)$, a lack of weight loss $(\mathrm{P}=0.03)$ and a lack of metastases $(\mathrm{P}=0.02)$ (Tables III and IV). Tumour diameter and total dose were not found to be prognostically significant. The mean total dose was 22.7 Gy [1 patient (3\%) received $11 \mathrm{~Gy}$ in 5 fractions, 3 patients (10\%) received 18 Gy in 6 fractions, 7 patients $(23 \%)$ received 20 Gy in 5 fractions 


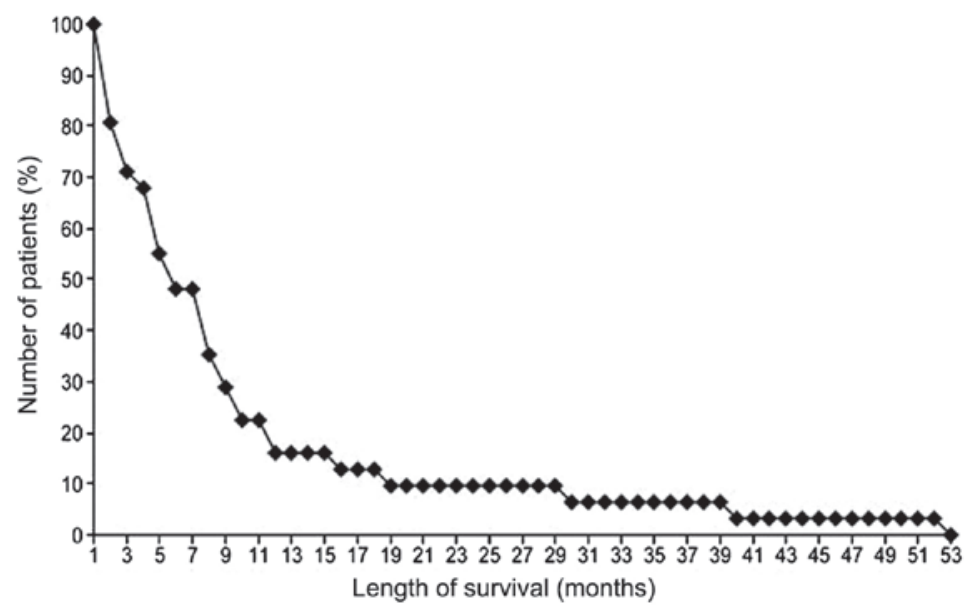

Figure 1. Survival rate analysis using the Kaplan-Meier survival curve in 31 pancreatic cancer patients.

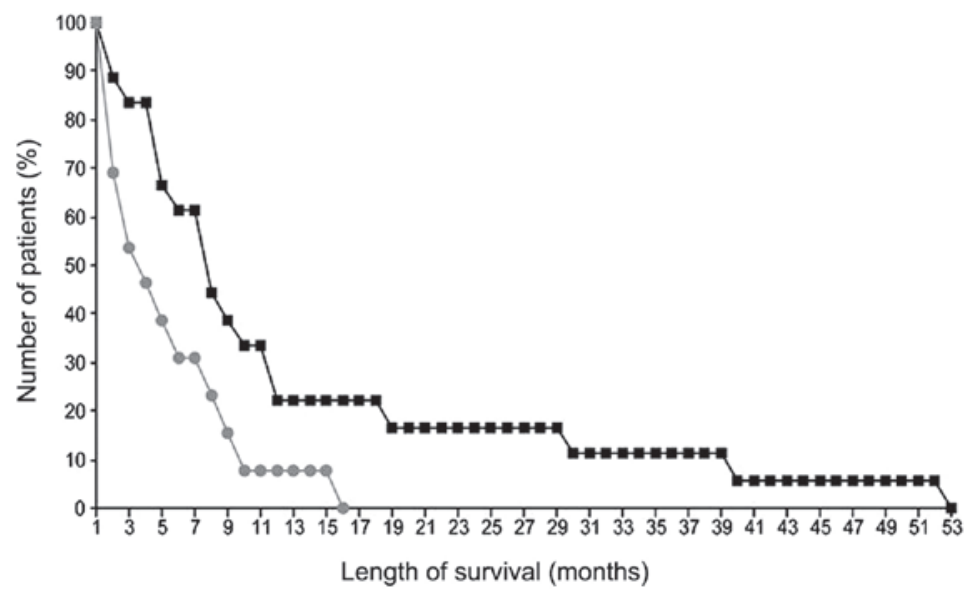

Figure 2. Kaplan-Meier survival curves of 18 men (squares) and 13 women (circles) who underwent palliative radiotherapy for pancreatic cancer (P=0.03).

and 19 patients $(61 \%)$ received 30 Gy in 10 fractions. A single patient received 6 Gy in 1 fraction].

The risk of premature death decreased by $\sim 5 \%$ with every increasing dose of RT $(\mathrm{HR}=0.947$; 95\% CI: 0.0208-0.0548; $\mathrm{P}=0.0083$ ).

\section{Discussion}

Most clinicians are cautious when recommending an active treatment modality for pancreatic cancer in their clinical practice, due to the poor prognosis of this disease, the wishes of the patients or advanced age, although patients may be in a reasonably good medical condition. Based on the results of Krzyżanowska et al (14) in a cohort study on locally advanced pancreatic cancer, $44 \%$ of the patients received some form of cancer-directed therapy [24\% received concurrent CRT therapy (CCRT), 13\% received RT alone and 7\% received chemotherapy alone]. According to that study, any type of active treatment was found to prolong survival.

Park et al (15), in a subgroup analysis of 340 patients with unresectable, locally advanced, or metastatic pancreatic cancer, found that stage III patients treated with either CCRT (median OS, 10.4 months) or chemotherapy alone (median OS, 11.3 months) exhibited a survival benefit over supportive care (median OS, 6.4 months), whereas stage IV patients treated with chemotherapy alone (median OS, 6.4 months) showed a survival benefit over supportive care (median OS, 3.1 months). The majority of patients who have pancreatic cancer in their initial evaluation have a combination of factors, such as advanced age, poor performance status, medical comorbidity or tumour-related conditions, such as anorexia; thus, they are poor candidates for aggressive therapy, such as CRT. It is important to perform a stratification of patients into treatment groups on the basis of prognostic factors $(16,17)$.

Morganti et al (18) evaluated 12 patients to observe whether a short RT treatment (30 Gy, 3.0 Gy/fraction) exhibited analgesic efficacy in patients with unresectable pancreatic carcinoma. They concluded that, in patients excluded from standard concomitant CRT, hypofractionated RT is feasible and results in pain relief in the majority of the patients. Our observations also included a reduced need for analgesics.

Pain due to pancreatic cancer is a manifestation of neural invasion and obstructive ductal physiological pain in nearly all patients. Intervention in the form of celiac neurolysis with chemical agents decreases pain (19) and may actually improve survival. Celiac neurolysis may be performed intraoperatively, percutaneously, or endoscopically under ultrasound guidance $(20,21)$. 
Other methods for reducing pain are advanced RT techniques, such as stereotactic body RT, which are associated with low rates of adverse effects and good local control in patients with locally advanced pancreatic cancer $(22,23)$. However, not all patients are good candidates for this type of sophisticated treatment.

The present study indicates that more active treatments should be attempted, even in cases of locally advanced unresectable pancreatic cancer. Palliative RT is a last resort to improve local pain control in patients with unresectable pancreatic cancer (24). A total of $30 \mathrm{~Gy}$ in 10 fractions is one of the most commonly used dose-fractionation regiments of palliative RT in cases with brain metastasis, bone metastasis and bleeding from advanced gastric cancer (25). Wong et al (26) compared the scheme of $30 \mathrm{~Gy}$ in 10 fractions and the scheme of $>30 \mathrm{~Gy}$ with concurrent infusions of 5-FU, and the median survival times were similar with both schemes. Considering the median survival time (5 months) following RT, this regimen appears to be adequate for patients with poor prognosis.

The classical endpoints of palliative treatment are survival, tumour response and quality of life. Patients who were treated with palliative RT gained clinical benefits. Undoubtedly, these treatments have reinforced the role of analgesic drugs.

Palliative treatment is often the only remaining option in the management of pancreatic carcinoma, but its efficacy is poor due to low tumour sensitivity and inadequate treatment protocols. There are several options for palliative treatment, with either an antitumor effect as immunotherapy $(27,28)$ or as supportive care. The present study demonstrated that patients with locally advanced pancreatic cancer who receive palliative RT have better survival rates compared with those who receive supportive care alone. RT, when used as a palliative treatment, was well-tolerated and was associated with a good median OS rate. Hence, palliative RT remains a non-invasive treatment option for improving pain control in patients with locally advanced or metastatic pancreatic cancer.

\section{References}

1. Heinemann V: Present and future treatment of pancreatic cancer. Semin Oncol 29 (Suppl 9): 23-31, 2002.

2. Goggins M: Molecular markers of early pancreatic cancer. J Clin Oncol 23: 4524-4531, 2005

3. Chua YJ and Cunningham D: Adjuvant treatment for resectable pancreatic cancer. J Clin Oncol 23: 4532-4537, 2005.

4. Real FX: A 'catastrophic hypothesis' for pancreas cancer progression. Gastroenterology 124: 1958-1964, 2003.

5. Nitecki SS, Sarr MG, Colby TV and van Heerden JA: Long-term survival after resection for ductal adenocarcinoma of the pancreas. Is it really improving? Ann Surg 221: 59-66, 1995.

6. Sohn TA, Yeo CJ,Cameron JL, Koniaris L, Kaushal S, Abrams RA Sauter PK, Coleman J, Hruban RH and Lillemoe KD: Resected adenocarcinoma of the pancreas-616 patients: Results, outcomes, and prognostic indicators. J Gastrointest Surg 4: 567-579, 2000.

7. Bilimoria KY, Bentrem DJ, Ko CY, Tomlinson JS, Stewart AK, Winchester DP and Talamonti MS: Multimodality therapy for pancreatic cancer in the U.S.: Utilization, outcomes, and the effect of hospital volume. Cancer 110: 1227-1234, 2007.

8. Sener SF, Fremgen A, Menck HR and Winchester DP: Pancreatic cancer: A report of treatment and survival trends for 100,313 patients diagnosed from 1985-1995, using the National Cancer Database. J Am Coll Surg 189: 1-7, 1999.

9. Crane CH, Evans DB, Wolff RA, Abbruzzese JL, Pisters PWT and Janjan NA: The pancreas. In: Radiation oncology: rationale, technique, results. 8th edition. Cox J and Ang KK (eds). Mosby, St Louis, MO, pp456-480, 2003.
10. Mantell BS: Radiotherapy for dysphagia due to gastric carcinoma. Br J Surg 69: 69-70, 1982.

11. Myint AS: The role of radiotherapy in the palliative treatment of gastrointestinal cancer. Eur J Gastroenterol Hepatol 12: 381-390, 2000.

12. Greene FL, Page DL, Fleming ID, Fritz AG, Balch CM, Haller DG and Morrow M: Digestive system. In: AJCC Cancer Staging Manual. 6th edition. Springer, Chicago, IL, pp151-157, 2002.

13. Cancer Therapy Evaluation Program: Common Terminology Criteria for Adverse Events, version 3.0. DCTD, NCI, NIH, DHHS. http://ctep.cancer.gov/protocolDevelopment/electronic_ applications/docs/ctcaev3.pdf. Accessed, August 9, 2006.

14. Krzyzanowska MK, Weeks JC, Earle CC Treatment of locally advanced pancreatic cancer in the real word: population-based practices and effectiveness. J Clin Oncol 21: 3409 3414, 2003.

15. Park JK, Yoon YB, Kim YT, Ryu JK, Yoon WJ and Lee SH: Survival and prognostic factors of unresectable pancreatic cancer. J Clin Gastroenterol 42: 86-91, 2008.

16. Rudra S, Narang AK, Pawlik TM, Wang H, Jaffee EM, Zheng L, Le DT, Cosgrove D, Hruban RH, Fishman EK, et al : Evaluation of predictive variables in locally advanced pancreatic adenocarcinoma patients receiving definitive chemoradiation. Pract Radiat Oncol 2: 77-85, 2012.

17. Wild AT, Ye X, Ellsworth SG, et al : The association between chemoradiation-related lymphopenia and clinical outcomes in patients with locally advanced pancreatic adenocarcinoma. Am J Clin Oncol 38: 259-265, 2015.

18. Morganti AG, Trodella L, Valentini V, Barbi S, Macchia G, Mantini G, Turriziani A and Cellini N: Pain relief with short-term irradiation in locally advanced carcinoma of the pancreas. J Palliat Care 19: 258-262, 2003.

19. Lillemoe KD, Cameron JL, Kaufman HS, Yeo CJ, Pitt HA and Sauter PK: Chemical splanchnicectomy in patients with unresectable pancreatic cancer. A prospective randomized trial. Ann Surg 217: 447-457, 1993.

20. Wong GY, Schroeder DR, Carns PE, Wilson JL, Martin DP, Kinney MO, Mantilla CB and Warner DO: Effect of neurolytic celiac plexus block on pain relief, quality of life, and survival in patients with unresectable pancreatic cancer: A randomized controlled trial. JAMA 291: 1092-1099, 2004.

21. Levy MJ and Wiersema MJ: EUS-guided celiac plexus neurolysis and celiac plexus block. Gastrointest Endosc 57: 923-930, 2003.

22. Huguet F, Goodman KA, Azria D, Racadot S and Abrams RA: Radiotherapy technical considerations in the management of locally advanced pancreatic cancer: American-French consensus recommendations. Int J Radiat Oncol Biol Phys 83: 1355-1364, 2012.

23. Goyal K, Einstein D, Ibarra RA, Yao M, Kunos C, Ellis R, Brindle J, Singh D, Hardacre J, Zhang Y, et al : Stereotactic body radiation therapy for nonresectable tumors of the pancreas. J Surg Res 174: 319-325, 2012.

24. Cascinu S, Falconi M, Valentini V and Jelic S; ESMO Guidelines Working Group: Pancreatic cancer: ESMO Clinical Practice Guidelines for diagnosis, treatment and follow-up. Ann Oncol 21 (Suppl 5): v55-v58, 2010.

25. Asakura H, Hashimoto T, Harada H, Mizumoto M, Furutani K, Hasuike N, Matsuoka M, Ono H, Boku N and Nishimura T: Palliative radiotherapy for bleeding from advanced gastric cancer: Is a schedule of $30 \mathrm{~Gy}$ in 10 fractions adequate? J Cancer Res Clin Oncol 137: 125-130, 2011.

26. Wong AA, Delclos ME, Wolff RA, Evans DB, Abbruzzese JL, Tamm EP, Xiong HQ, Ho L and Crane CH; Pancreatic Tumor Study Group: Radiation dose considerations in the palliative treatment of locally advanced adenocarcinoma of the pancreas. Am J Clin Oncol 28: 227-233, 2005.

27. Royal RE, Levy C, Turner K, Mathur A, Hughes M, Kammula US, Sherry RM, Topalian SL, Yang JC, Lowy I, et al : Phase 2 trial of single agent ipilimumab (anti-CTLA-4) for locally advanced or metastatic pancreatic adenocarcinoma. J Immunother 33: 828-833, 2010.

28. Le DT, Lutz E, Uram JN, Sugar EA, Onners B, Solt S, Zheng L, Diaz LA Jr, Donehower RC, Jaffee EM, et al : Evaluation of ipilimumab in combination with allogeneic pancreatic tumor cells transfected with a GM-CSF gene in previously treated pancreatic cancer. J Immunother 36: 382-389, 2013. 